\title{
HEART-VECTOR AND LEADS. PART II
}

BY

\author{
H. C. BURGER AND J. B. VAN MILAAN
}

From the Physisch Laboratorium der Rijks Universiteit te Utrecht, Holland

Received May 27, 1947

In a preceding paper (Burger and van Milaan, 1946) we have treated the problem of the relation between the heart-vector and the leads that are usual in electrocardiography. Therein we pointed out that this relation is given by three linear equations, characterized by nine coefficients. The value of these coefficients was determined by means of a model of the human body, a glass phantom filled with an electrolytically conducting liquid. An artificial heart was used to put up an electric current field in the liquid, and the potential differences caused by this field were measured at the extremities $L, R$, and $F$.

The human body, however, is not a homogeneous conductor and especially, the lungs, the spinal column, and the liver cause a lack of homogeneity. Further, not only the leads from the extremities are of interest, but the increasing importance of the præcordial leads made it necessary to extend the method to other electrodes as well as to the extremities. The component of the heart vector, directed horizontally from the back to the chest (Y-component) is in this case of preponderating importance. We shall describe an improvement of our former method, allowing for the points mentioned above.

\section{The New Phantom}

To be able to install the internal organs and to fix the electrodes at different points on the surface of the body, the phantom had to be constructed in such a way that its inner side was within reach. It was of michaplast, that had formed the cover of a small statue of a human being on a scale of $1: 3$. So it had the normal proportions of a true human body. It was cut into two pieces by a plane parallel to the frontal plane of the body. Of the two pieces the chest-side was the larger one and formed a small basin (Fig. 1) standing on the table, the phantom lying face downward. Arms and legs of the phantom were only short stubs, as the current does not penetrate in them. This part was placed in a large basin, filled with a solution of copper sulphate. The back-side formed a cover, in which holes were drilled to let the air escape. A broad flange prevented the current-field from leaking to the outside.

The artificial heart $H$ consisted of two parallel copper plates of $1.0 \mathrm{~cm}$. diameter and 1.0 $\mathrm{cm}$. apart. The space between was filled with an insulating substance (Burger and van Milaan, 1946, p. 159). It was fixed to a glass tube $T$, that protruded from the back-cover of the phantom. One heart, with its axis perpendicular to the glass tube, was turnable on a vertical axis, a dial $\mathrm{D}$ indicating its direction. Herewith it was possible to set up the component of the heart-vector, perpendicular to the median plane of the body (X-component), or of the component in the direction of its length-axis (Z-component). Another heart gave the component, vertical in the phantom (Y-component). Either heart was placed in the normal position. A potential difference of 4 volts set up the current field inside the phantom.

In addition to the extremity electrodes, $L, R$, and F, we have used a series of electrodes in different places. Pieces of copper foil of $1-0.5 \mathrm{sq} . \mathrm{cm}$., connected to an insulated wire, were fixed to the inner wall of the phantom in the desired position. The potential difference 


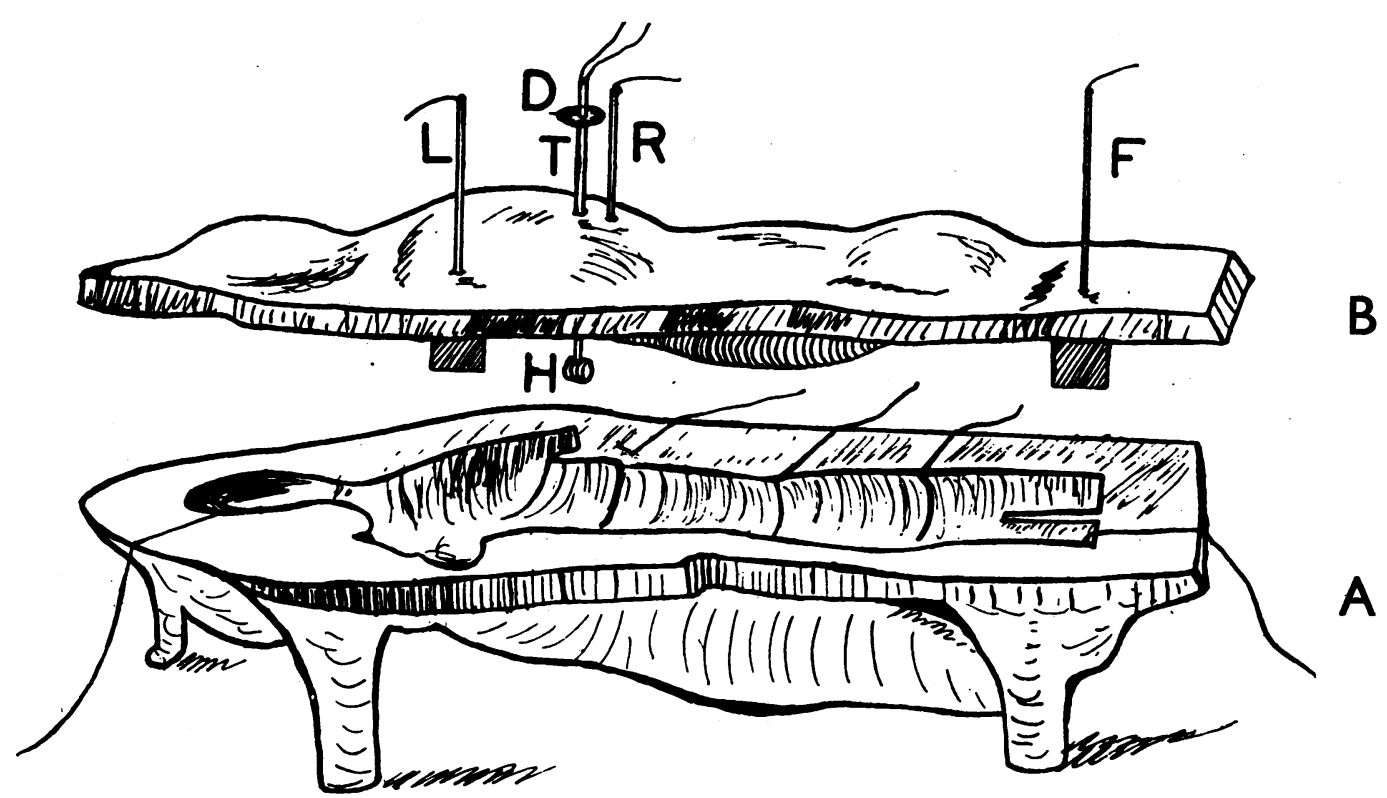

FIG. 1.-Phantom to measure the coefficients in the relation between heart-vector and leads.

(A) chest-side.

L, R, and F: extremity electrodes.

(B) back-side.

H: artificial heart with protruding glass tube (T) with dial (D).

between two electrodes was measured by means of a galvanometer provided with a high resistance.

We have asked if the back-cover was essentially necessary. Indeed it proved to be that the potential differences were of an entirely different value if the back-cover was omitted, the heart being placed in the same position. Even if the back-cover was elevated only a few millimetres, the change was perceptible. So we must conclude that measurements with an open basin cannot have any quantitative significance (Guckes, 1939; Hess, 1935; Hollman and Hollmann, 1937; Molz, 1939; and Schellong, 1939).

The lungs in the human body must have a great influence on the current-field of the heart, as their specific resistance is so much greater than that of the other parts of the body: this is a consequence of the air they contain. From anatomical data of the volume of the lungs and physiological data of their air content we have roughly calculated their specific resistance, and that in the state of maximal inspiration, maximal expiration, and in the normal condition. The specific resistance of the lung tissues was supposed to be the same as the average of the rest of the trunk. The result was that the lungs conduct about four times less than that rest.

This ratio can be verified as follows. Formerly we have measured the mean specific resistance of the trunk in the above-mentioned three states of the lungs (Burger and van Milaan, 1943). These specific resistances can be calculated approximately from anatomical date of the trunk, accepting the value of the specific resistance of the lungs, mentioned above.

The substance of which the artificial lungs have to be made must have a specific resistance, four times greater than that of the surroundings. We have found that sand in an electrolytical solution fulfils this requirement. We have, therefore, made two bags of cotton, the shape of which was, as accurately as possible, that of (real) human lungs. They were filled with sand and at their inner sides we have modelled to the best of our ability the hollows for the heart with needle and thread. It was, however, not possible to give them at the foot side the excavation of the pleural dome. The lungs were placed in the chest-part of the phantom. 
Some nipples, fixed to its wall, kept the lungs at some distance from it. In this way a conducting layer was left free, just as is the case between the human lungs and the chest wall.

The artificial liver was made in the same way as the lungs. The liver, however, seems to have a specific resistance, depending greatly on circumstances, namely on the fat and blood contents. Our measurements show that the influence of the liver on the relation between heart-vector and leads is only small.

Much greater is the influence of the spine, as it consists of badly conducting bone. The artificial spine was made of cork and was fixed to the back-cover. We have not tried to give it exactly the right shape, but we have given it the average cross-section of the true spine (on a scale $1: 3$ ).

\section{RESULTS}

The results of our measurements are all given in absolute units just as in our preceding paper. They hold good for a human body of average dimensions. The potential difference (lead) is expressed in volts, the heart-vector in volts $\times \mathrm{cm}^{2}$. The coefficients, therefore, in the equation:

Lead $=\mathrm{aX}+\mathrm{bY}+\mathrm{cZ}(\mathrm{X}, \mathrm{Y}$, and $\mathrm{Z}=$ components of the heart vector $)$ are expressed in volt/volt $\mathrm{cm}^{2}=\mathrm{cm}^{-2}$. They are inversely proportional to the dimensions of the body.
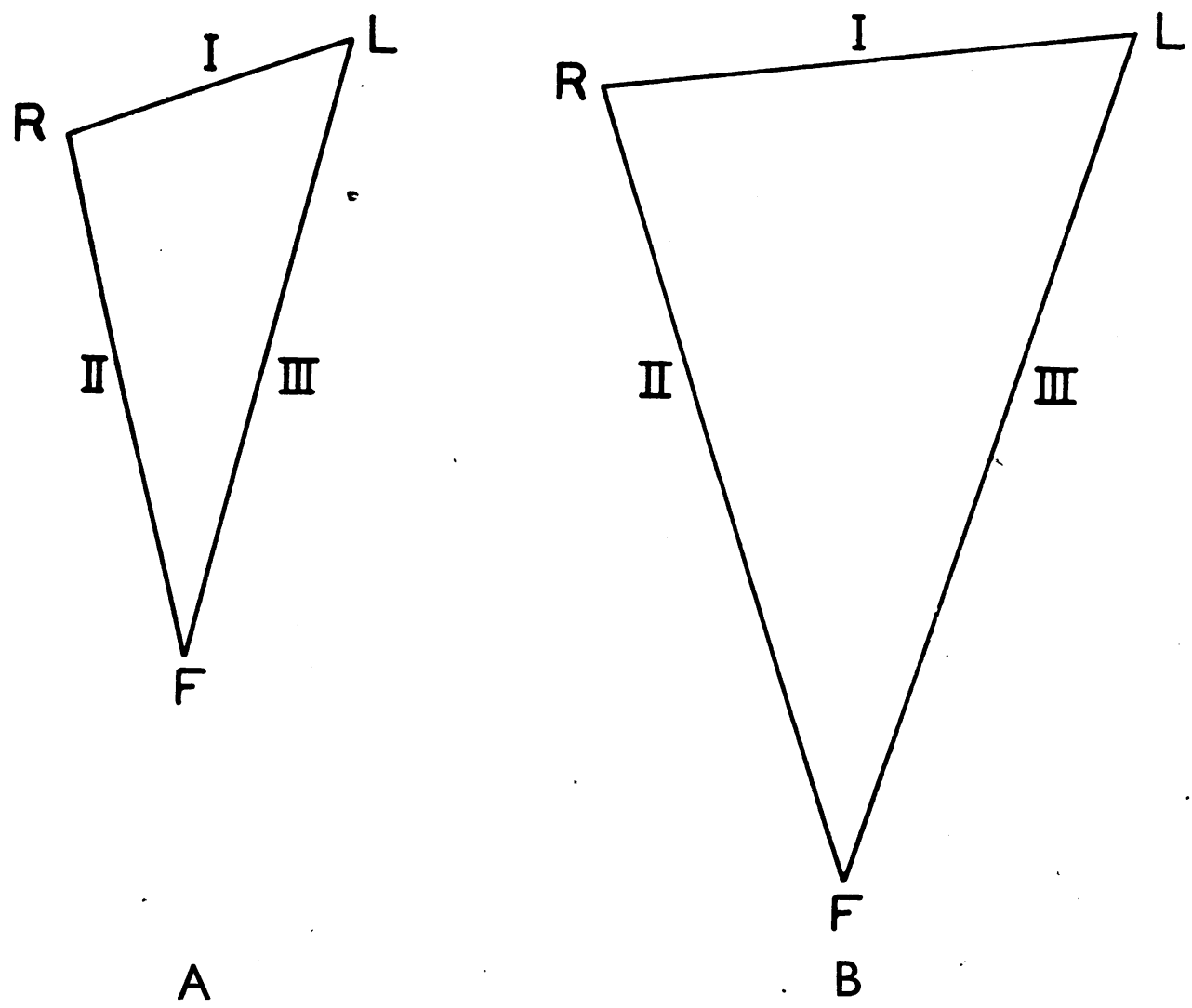

Fig. 2.-(A) Triangle, representing the relation of heart-vector and leads, deduced from a phantom with lungs and spine. (B) The same, without lungs and spine. 
The measurement is performed in using an artificial heart giving the components $\mathrm{X}, \mathrm{Y}$, and $\mathrm{Z}$ one by one. Each time a lead is measured, and from the value of lead and component the corresponding coefficient is to deduce as quotient of lead and component.

\section{Limb Leads}

First we have checked our method by measurements of the limb leads of the phantom without internal organs. The result was in accordance with our preceding measurements with the glass phantom.

The influence of the lungs was very marked. The contribution of the horizontal component (X) to lead I especially was reduced appreciably by the lungs. This was to be expected, as the badly conducting lungs at both sides of the heart screen off the current in a direction from the right arm to the left one. The spine did not alter the ratio of the leads appreciably but their absolute values were increased. The liver altered the leads so slightly, that, as a rule, we have omitted it.

The numerical result with the phantom, provided with lungs and spine, is given in the. following formulæ:

$$
\left.\begin{array}{rl}
I & =(65 \mathrm{X}-21 \mathrm{Z}) \times 10^{-5} \\
\mathrm{II}^{*} & =(-25 \mathrm{X}-120 \mathrm{Z}) \times 10^{-5} \\
\mathrm{III} & =(-40 \mathrm{X}+141 \mathrm{Z}) \times 10^{-5}
\end{array}\right\}
$$

The mathematical consequence of this analytical relation between heart-vector and leads is that it may be represented geometrically. In Fig. $2 \mathrm{~A}$ we give this representation, coming in place of the well-known triangle of Einthoven. Fig. 2B represents the relation between leads and heart-vector as found with the phantom without internal organs. The leads I, II, and III are proportional to the product of the projection of the heart-vector on the sides of this triangle and the length of the side on which it is projected.

\section{Pracordial Leads}

Up to now we have treated the problem as a two-dimensional one. Only the components $\mathrm{X}$ and $\mathrm{Z}$ parallel to the frontal plane, were taken into account. But as a matter of fact the problem is a three-dimensional one (Schellong, 1939); the heart-vector has also a Y-component, horizontal and parallel to the medium-plane. We will call it positive if it is directed from back

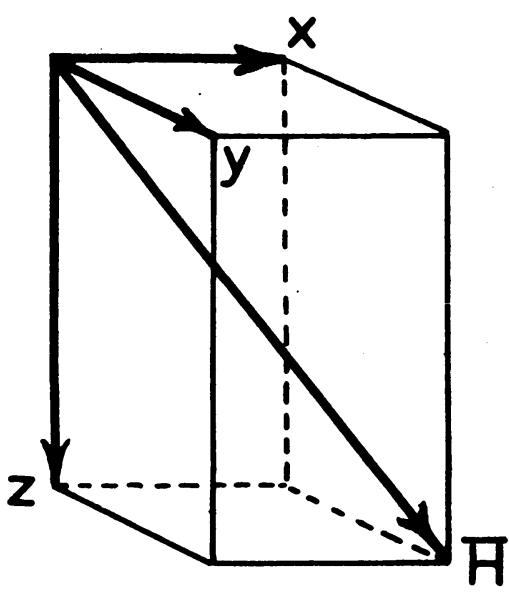

FIG. 3.-Heart-vector $\overline{\mathrm{H}}$ and its rectangular components $\mathrm{X}$, $\mathbf{Y}$, and $\mathbf{Z}$. to chest. Fig. 3 gives the situation of the three components of the heart-vector.

To find all the three components $\mathrm{X}, \mathrm{Y}$, and $\mathrm{Z}$ of the heart-vector we need three equations, so we have to measure three independent leads. The extremity leads I, II, and III cannot serve this purpose, as they are dependent, their sum being zero. To get the three independent equations necessary to determine unambiguously the three components of the heart-vector, at least one more electrode has to be used. As such an electrode one of the usual præcordial leads (IV) is suitable. As the position of lead IV we have taken a point in the mammary line and in the fifth intercostal space. We have measured the potential difference be tween IV and the left foot (F). It appeared to be:

$$
\mathrm{IV}=(19 \mathrm{X}+30 \mathrm{Y}+20 \mathrm{Z}) \times 10^{-5} \quad \text {. . . (3) }
$$

This equation must be combined with two equations for the leads I and II, in which the component $Y$ is

\footnotetext{
* For reasons of symmetry lead II is given the opposite sign to the usual.
} 
not omitted. These equations are obtained by putting in the equation (2) the term with Y, provided with its coefficient, found with the phantom. These equations are:

$$
\left.\begin{array}{rl}
\mathbf{I} & =(65 \mathrm{X}-17 \mathrm{Y}-21 \mathrm{X}) \times 10^{-5} \\
\mathbf{I} & =(-25 \mathrm{X}-15 \mathrm{Y}-120 \mathrm{Z}) \times 10^{-5}
\end{array}\right\} \quad . \quad . \quad . \quad . \quad . \quad .
$$

From the three equations ( 3 ) and (2A), it is possible to solve $X, Y$, and Z. The result is:

$$
\left.\begin{array}{l}
X=(12.9 \mathrm{I}-1.2 \mathrm{II}+6.4 \mathrm{IV}) \times 10^{2} \\
\mathbf{Y}=(-7.0 \mathrm{I}+6.4 \mathrm{II}+32.8 \mathrm{IV}) \times 10^{2} \\
Z=(-1.8 \mathrm{I}-9.0 \mathrm{II}-5.6 \mathrm{IV}) \times 10^{2}
\end{array}\right\}
$$

Using these coefficients, one can calculate the components $\mathrm{X}, \mathrm{Y}$, and $\mathrm{Z}$ of the heart-vector from the simultaneously measured leads I, II, and IV.

The equations (2), in which the Y-component is omitted, give an erroneous result for the $\mathrm{X}$ - and $\mathrm{Z}$-components, that we will call $\mathrm{X}^{\prime}$ and $\mathrm{Z}^{\prime}$. If $\mathrm{X}$ and $\mathrm{Z}$ are the true values of these components, we can prove that:

$$
\begin{aligned}
& X^{\prime}=X-0 \cdot 21 Y \\
& Z^{\prime}=Z+0 \cdot 17 Y
\end{aligned}
$$

We see, therefore, that the omission of $\mathrm{Y}$ gives a rather satisfactory value of the projection of the heart-vector, if its $\mathrm{Y}$-component is smaller than $\mathrm{X}$ and $\mathrm{Z}$.

Neglecting the Y-component, from the equations (2) we can deduce an approximate value

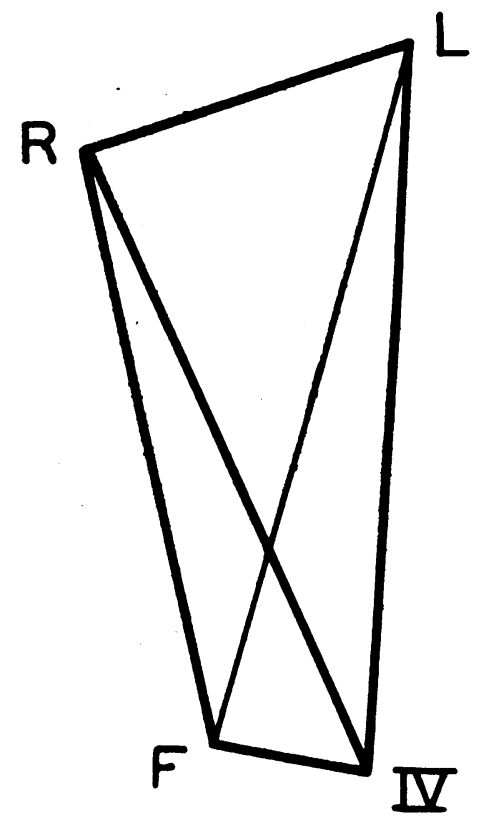

FIG. 4.-Tetrahedron, representing the relation of heartvector and leads. of the absolute magnitude of the heart-vector during its maximum (R). Assuming for I a value of $1 \mathrm{mV}$ and for II a value of $-1.5 \mathrm{mV}^{*}$ ) we find that the maximal $\mathrm{R}$-deflection corresponds to a heart-vector of about 2 volt $\mathrm{cm}^{2}$.

Just as in the two-dimensional case, it is possible in the three-dimensional one to give a geometrical representation. Instead of a triangle we have to work with a tetrahedron, the angular points of which correspond to right arm (R), left arm (L), left foot (F), and præcordial electrode (IV) (Fig. 4). Each lead is the product of the projection of the heart-vector on the corresponding edge multiplied by the length of that edge. So if $p$ is the projection of the heartvector $\bar{H}$ on the edge $R L$, lead $I$ is equal to $p \times R L$ etc. The shape of the tetrahedron can be deduced easily from the coefficients of the equations (3) and (2A), giving the leads as a function of the components $\mathrm{X}, \mathrm{Y}$, and $\mathrm{Z}$ of the heart-vector.

To solve the problem of the relation between heart-vector and leads completely, it would be necessary to measure the potential-difference between all the points on the surface of the phantom and a fixed point e.g. the left foot (F), and for the three components of the heart-vector. We do not know if there are leads that are to be preferred essentially above I, II, and IV, nor do we know if there are arguments from the clinical side for using other ones.

We have made some measurements with præcordial electrodes in the median-plane. The origin of the potential-differences was the Z-component of the heart-vector having unit value. Fig. 5 gives the potential difference between these electrodes and $F$, as a function of their distance

* As II has the opposite sign to usual, it has to be taken as negative. 


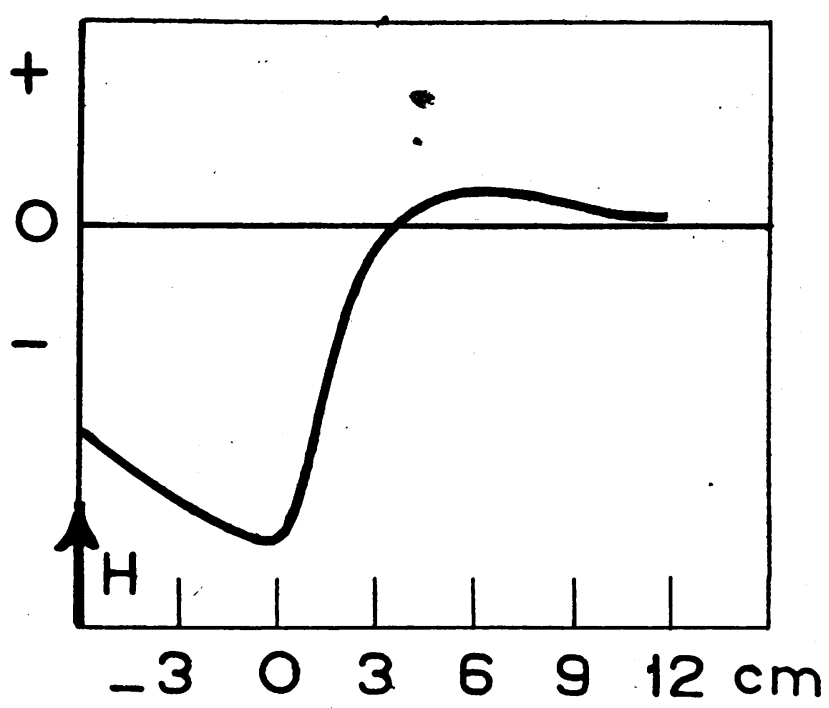

FIG. 5.-Potential difference between precordial electrodes in the median plane and the left foot $(F)$. The numbers give the distance below the axillary line in the phantom. $\mathbf{H}=$ head.

below the axillary line. The point, denoted by $\mathrm{H}$, represents the head, inside which the potentialdifferences can be neglected. The general type of the curve can be explained theoretically.

\section{Leads that depend on only one Component of the Heart-Vector}

In equation (1) the coefficients $a, b$, and $c$ depend on the position of the two electrodes. It is possible to choose these in such a way that $b$ and $c$ both are zero. Then the lead is only depending on $\mathrm{X}$; it is proportional to $\mathrm{X}$ : Lead $=\mathrm{aX}$. In the same way it can be arranged that the leads are depending only on $\mathrm{Y}$ and only on $\mathrm{Z}$. Only with a phantom is it possible to find positions of the electrodes that fulfil these requirements.

$\mathrm{X}$-lead. The electrodes are placed respectively in the side, $12 \mathrm{~cm}$. below the axilla and on the right arm. X-lead $=1.56 \times 10^{-3} \mathrm{X}$.

Y-lead. The one electrode is placed on the chest wall, $9 \mathrm{~cm}$. to the left of the median plane and $27 \mathrm{~cm}$. below the axilla; the other is placed on the back in the median plane, $27 \mathrm{~cm}$. below the axilla. Y-lead $=2.4 \times 10^{-3} \mathrm{Y}$.

Z-lead. The electrodes are placed respectively on the left leg or foot (F) and on the throat $6 \mathrm{~cm}$. to the right of the median plane, at the height of the point of the larynx; Z-lead $=1 \cdot 0^{9} \times 10^{-3} \mathrm{Z}$.

Only by experience can it be decided if these leads have any practical value,

\section{Short-circuiting of the Electromotive Action of the Heart by the Blood}

The value of the heart-vector, deduced by the above method is only an effective one. The electromotive field strength of the heart muscle causes a current not only in the tissue surrounding it but also in the blood inside. As the latter is the better conducting material it acts as a shunt, the outer current being diminished by it (Beleradek and Noyons, 1923): The value, therefore, that is found for the heart-vector is too small. From our measurements with the phantom it is impossible to find the true heart-vector. The construction of the artificial heart leaves this complication out of account. 


\section{SUMMARY}

The phantom used for the determination of the relation between heart-vector and leads has been improved. It has been provided with lungs and a spine and it is possible to apply electrodes in any desired position. The influence of lungs and spine is appreciable.

The results̀ of the measurements with lead IV and other præcordial leads are given.

It is possible to find leads that depend on only one component of the heart-vector.

\section{REFERENCES}

Beleradek, J., and Noyons, A. K. M. (1923). C. R. Soc. Biol., Paris, 88, 621.

Burger, H. C., and van Milaan, J. B. (1943). Acta med. Scand., 114, 584.

- (1946). Brit. Heart J., 8, 157.

Guckes, E. (1939). $\quad Z$. Exp. Med., 104, 705.

Hess, W. (1935). Z. Kreigslaufforschung, 27, 433.

Hollmann, H. E. and Hollmann, W. (1937). Z. Instr. Kunde, 57, 285.

Molz, B. (1939). Pflüg Arch. ges. Physiol., 242, 416.

Schellong, F. (1939). Grundzüge einer klinischen Vektordiagraphie des Herzen, Berlin, Springer. 